\title{
A Method of Leak Detection for Spacecraft on-orbit based on Acoustic Emission
}

\author{
L. QI , D.H. MENG, R.X. YAN, L.C. SUN, Y. WANG, W. SUN, R.P. SHAO, W.D. LI, Z. LI \\ Beijing Institute of Spacecraft Environment Engineering, Beijing, China
}

\begin{abstract}
With the development of spaceflight activity of human, the number of space debris has increased dramatically. Spacecraft will be impacted by large amount of small debris in space during flight. In order to detect the gas leak in time, a new method of leak detection for spacecraft on-orbit based on acoustic emission is important. Gas leak detection technology based on the acoustic emission in the field of manned space flight has been successfully applied. In this paper, some of the latest research is introduced giving some reference to leak detection for spacecraft on-orbit. Gas leak produces a large number of acoustic emission signal, and the frequency of the signal covers multiple frequency bands. The smaller the leakage is, the smaller the signal amplitude is, but the more high frequency signal component is contained. In normalized energy distribution map, different aperture leakage acoustic emission signals have a certain similarity, but have obvious difference with pump noise signal whose frequency hand is mainly below $20 \mathrm{kHz}$.
\end{abstract}

KEYWORD: Acoustic emission; Spacecraft on-orbit; Leak detect; Average frequency spectrum

\section{INTRODUCTION}

With the development of space science and technology, a large number of micro meteor as well as space debris has become the main threat to the manned spacecraft[1-6]. Once the space debris distributed between $200 \mathrm{~km}$ and $800 \mathrm{~km}$ collide with the spacecraft, gas leak may occur, payload cannot work normally, and lives of the astronauts may be threatened. In order to guarantee the normal operation of the spacecraft and lives of the astronauts, to strengthen its own security protection for spacecraft on-orbit is very important, and gas leak detection has become a problem for us to seriously study.

\section{PRINCIPLE}

Based on gas dynamic acoustics theory, sealing structure once occurs gas leak, high-speed airflow from the leakage becomes flow turbulent jet, inspiring a lot of sound wave signal as part of the jet noise. Gas leak acoustic emission signal spreads in the outer wall and converts to electrical signal by piezoelectric acoustic emission sensors. Acoustic emission signal is collected for leak detection and leak evaluation based on waveform characteristic parameter analysis.
Waveform characteristic parameter analysis method is a common signal processing method used in acoustic emission detection. Typical acoustic emission waveform characteristic parameters such as rise time, amplitude, RMS are the main basis of the method analysis. Because gas leak inspires continuous acoustic emission signal, wave hit, the most value and average amplitude are the useful characteristic parameters. Acoustic emission signal is a typical non-stationary signal, so single acoustic emission signal characteristic parameter is contingent. This paper introduces a method based both on feature of acoustic emission waveform parameter analysis and average spectrum analysis. Acoustic emission signal is transformed to frequency spectrum by fast Fourier transform. The average spectrum is calculated, as shown in the type (1). $S$ is on behalf of the acoustic emission signal excited condition, $N_{s}$ is the sample size for leakage signal.

$X_{\mathrm{S}}(\omega)=\frac{\sum_{i=1}^{N_{\mathrm{S}}} \int_{0}^{T} x_{\mathrm{S}, i}(t) e^{-j \omega t} d t}{N_{\mathrm{S}}}$

Used inverse Fourier transform, the average acoustic emission signal in time domain is got as shown in type (2):

$x_{\mathrm{S}, \text { mean }}(t)=\frac{1}{2 \pi} \int_{-\infty}^{\infty} X_{\mathrm{S}}(\omega) e^{j \omega t} d \omega$ 
The method can overcome the single signal randomness, reduce the effect of random noise, improve signal-to-noise ratio of the acoustic emission signal effectively and make the analysis more statistically significant.

Wavelet packet analysis is an effective approach for the analysis of non-stationary signal, compared with wavelet transform. In this paper, background pump noise and different acoustic emission signal inspired by different aperture leakage are collected and analyzed. By research the average time domain waveform under different leakage state, wavelet package analysis of characteristics of normalized energy band are given. At last, wavelet packet frequency band is adjusted according to the frequency from low to high.

\section{EXPERIMENT}

\subsection{Experiment platform}

The experiment platform (seen in fig. 1) including:

1. Gas leak experiment platform: $1 \mathrm{~m} * 1 \mathrm{~m}$ square platform made in 304 stainless steel is used, in which there are three different circular leakage apertures of $1 \mathrm{~mm}, 1.5 \mathrm{~mm}, 2 \mathrm{~mm}$. Besides, gas leak experimental platform includes vacuum pumps, vacuum bellows and digital display type vacuum gauge, etc.

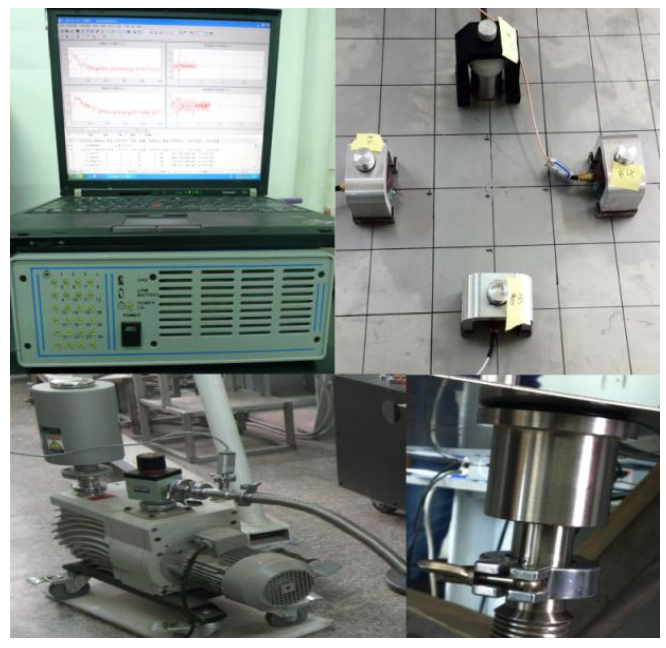

Figure.1 Gas leak experiment platform

2. Acoustic emission detection system: it contains physical acoustics corporation (PAC) $\mu$ Samos type digital multi-channel acoustic emission detection system, whose parameters is shown in table 1 .

Table. 1 Parameters of $\mu$ Samos system

\begin{tabular}{|l|l|}
\hline parameter name & parameter values \\
\hline sampling precision & 16 bit \\
\hline sampling frequency $/ \mathrm{kHz}$ & 3000 \\
\hline sensor model & wsa \\
\hline sensor frequency $/ \mathrm{kHz}$ & $5 \sim 1000$ \\
\hline gain $/ \mathrm{dB}$ & 40 \\
\hline
\end{tabular}

\subsection{Gas leakage acoustic emission signal acquisition}

Considering frequency band of the gas leak acoustic emission signal is wide, wsa sensor is used to receive the acoustic emission signal.

With coupling agent, acoustic sensor are fixed on the 302 stainless steel plate, the gain of the preamplifier is set to $40 \mathrm{~dB}$, Hsu - Nielsen break is used before formal acquisition to verify the performance of the acoustic emission sensors and background noise level.

Use the vacuum pump to pump air into vacuum state. When the value of type vacuum gauge is stable, differential pressure between both ends of leakage is stable. Make the acquisition of the acoustic emission signal under different leakage state, leakage acoustic emission signal samples including vacuum pump running background noise, and acoustic emission signal excited by $1 \mathrm{~mm}, 1.5 \mathrm{~mm}$ and $2 \mathrm{~mm}$ aperture leakage.

\section{DATA PROCESS}

In order to meet the requirements of online testing, acoustic emission leak detection system needs to overcome the influence of pump running noise. Therefore, the pump running noise is obtained and analyzed, and the average time domain waveform and the average frequency spectrum are shown in figure 2 .

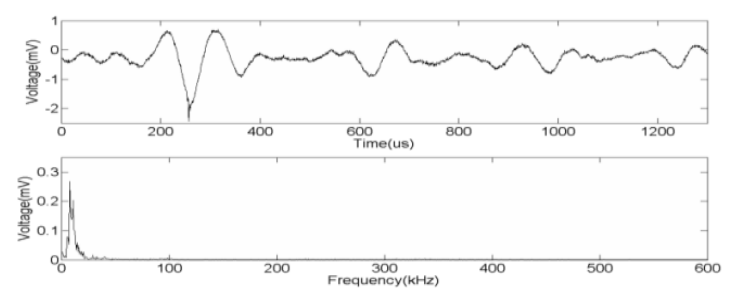

Figure.2 Average acoustic emission waveform and frequency spectrum of the pump running noise

From figure 2, frequency of pump running noise is relatively single, mainly distributed in below 20 $\mathrm{kHz}$, which belongs to the typical low frequency signal in the field of acoustic emission. So a real-time high-pass filter is need to eliminate pump running noise and improve the signal-to-noise ratio.

The acoustic emission signals inspired by three different aperture of leakage are collected and analyzed. Average time domain waveform and frequency spectrum are shown in figure 3 . 

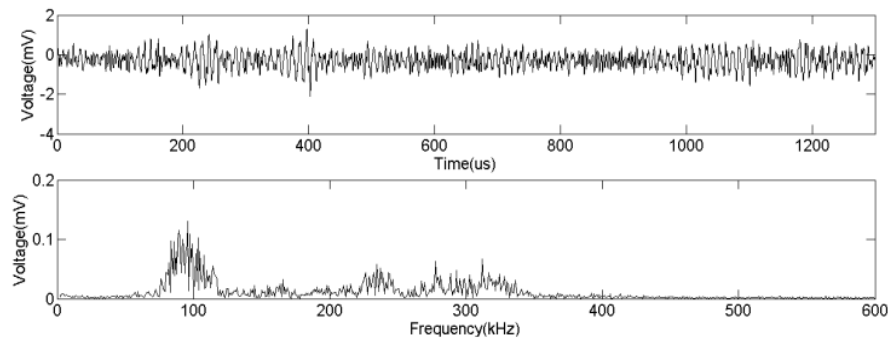

(a) $1 \mathrm{~mm}$ 漏孔
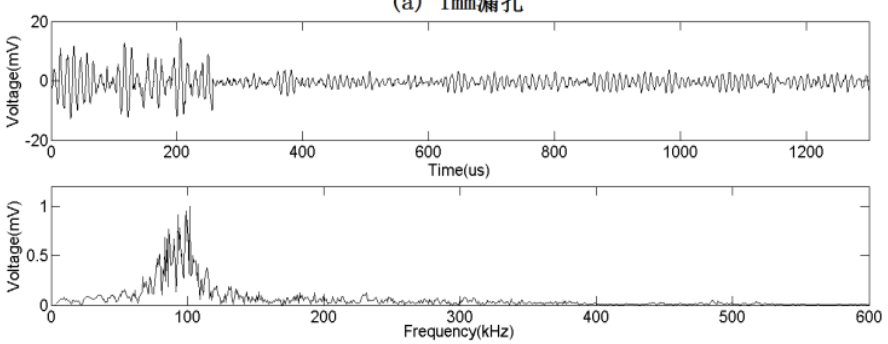

(b) $1.5 \mathrm{~mm}$ 漏孔
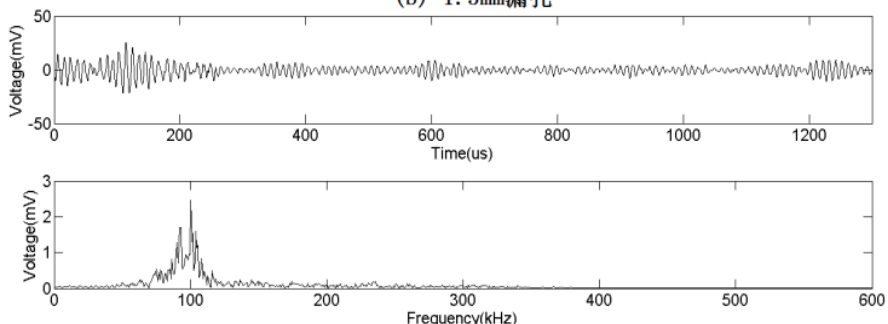

(c) $2 \mathrm{~mm}$ 漏孔

Figure.3 Average acoustic emission waveform and frequency spectrum of three different leak apertures

From figure 3, the acoustic emission signals inspired by three different aperture leakage average are similar both in time domain and frequency spectrum, but there are also some differences. First, along with the increase of leakage aperture, the amplitude of the leak acoustic emission signal increases significantly, from $1.5 \mathrm{mV}$ when leakage size is $1 \mathrm{~mm}$ to $15 \mathrm{mV}$ when leakage size is $1.5 \mathrm{~mm}$ and $25 \mathrm{mV}$ when leakage size is $2 \mathrm{~mm}$. It shows that under the same experiment conditions, the larger the leakage size is, the greater the acoustic emission signal amplitude is. Using this conclusion, leak is detected and the leakage size is estimated. Second, the acoustic emission signals inspired by three different aperture leakage have near main component when frequency is 100 $\mathrm{kHz}$. When the leakage aperture is $1 \mathrm{~mm}, 200 \mathrm{kHz}$ to $350 \mathrm{KHZ}$ high frequency acoustic emission signal is also inspired. The characteristic parameters of acoustic emission signals inspired by three different leakage are showed in table 2 .

Table. 2 Characteristic parameters of three different leak apertures

\begin{tabular}{|l|l|}
\hline leakage aperture & average signal amplitude \\
\hline $1 \mathrm{~mm}$ & $5.9 \mu \mathrm{V}$ \\
\hline $1.5 \mathrm{~mm}$ & $27.2 \mu \mathrm{V}$ \\
\hline $2 \mathrm{~mm}$ & $37 \mu \mathrm{V}$ \\
\hline
\end{tabular}

The characteristics of signals under different conditions are analyzed by wavelet packet energy nor- malization. The sampling frequency is $3 \mathrm{MHZ}$, the number of wavelet packet layer is 5 , and each frequency bandwidth of $46.875 \mathrm{kHz}$. Considering the frequency does not exceed the limit of $500 \mathrm{kHz}$, so normalized energy characteristics of first twelve bands is analyzed(seen in fig.4).
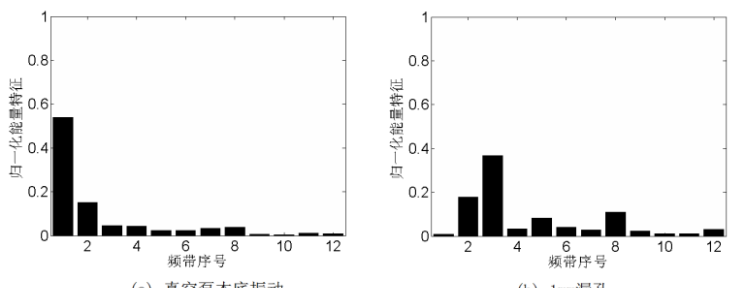

(a) 真空意本底振动
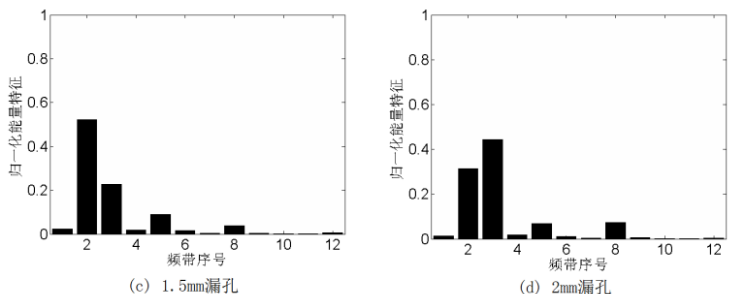

Figure.4 Normalized wavelet packet energy distributions of four states

From figure 4, the energy of pump noise signal is concentrated in the first two bands, and the energy in first frequency band accounts for about $53.93 \%$ of the total energy. Gas leak acoustic emission signal forms multiple energy distribution in frequency, and the energy in the second, three, five, eight frequency bands is greater. Characteristics of different gas leak acoustic emission signals have a certain similarity. Combined with the average spectrum of gas leak acoustic emission signal, the smaller the leakage is, the smaller the signal amplitude is, but the more high frequency signal component is contained.

Gas leak produces a large number of acoustic emission signal, and the frequency of the signal covers multiple frequency bands. In low frequency band, acoustic emission signal is commixed with the pump noisy, not suitable for leakage evaluation, so the high frequency part of leakage acoustic emission signal has more research value.

\section{CONCLUSION}

Gas leak detection technology based on the acoustic emission in the field of manned space flight has been successfully applied[7-10]. In this paper, some of the latest research is introduced giving some reference to leak detection for spacecraft on-orbit.

1. Gas leak produces a large number of acoustic emission signals, and the frequency of the signal covers multiple frequency bands. The smaller the leakage is, the smaller the signal amplitude is, but the more high frequency signal component is contained. 
2. In normalized energy distribution map, three different aperture leakage acoustic emission signals have a certain similarity, but have obvious difference with pump noise signal.

\section{REFERENCES}

[1] Rodriguez H M, Liou J-C, Orbital debris; past, present, and future. Orbital Debris Quarterly News. 2008, 12(3).

[2] J. Holliman."Leak in Mir may have been Located". in CNN Interactive Online, October, 3, 1997.

[3] C. V. D. Berg, "6th Spacewalk (EVA) Crew 24th Main Expedition MIR," in MirNews, vol. 402, January 8, 1998.

[4] Officially cataloged by U.S. Space surveillance network, Monthly number of objects in earth orbit by object type. Orbital Debris Quarterly News. 2009, 13(1).

[5] G Stansbery, Growth in the number of SSN tracked orbital objects. Orbital Debris Quarterly News. 2004, 8(1).
[6] Liou J-C, Johnson N L, Hill N M, Stabilizing the future LEO debris environment with active debris removal. Orbital Debris Quarterly News. 2008, 12(4).

[7] Guo H W, Shi W K, Deng Y. Evaluating sensor reliability in classification problems based on evidence theory. IEEE Transactions on Systems, Man and Cybernetics, Part B, 2006, 36(5):970981.

[8] Kevin D. Champaigne, Jonathan Sumners. Wireless impact and leak detection and location systems for the ISS and shuttle wing leading edge. IEEE Aerospace Conference Proceedings Proceedings.2005: 1-7.

[9] Kevin Champaigne and Eric Krug. Wireless Instrumentation System for Plasma Measurements on the International Space Station. AIAA Paper 2002-0937, AIAA Aerospace Sciences Meeting \& Exhibit, 40th, 2002.

[10] Stephen D. Holland, Ronald A. Roberts, Dale E. Chimenti. Leak detection in spacecraft using structure-borne noise with distributed sensors. APPLIED PHYSICS LETTERS. 2005(86):1-4. 